\title{
ANN-Based Forecasting of Hydropower Reservoir Inflow
}

\author{
Antans Sauhats, Roman Petrichenko, Zane Broka, Karlis Baltputnis, Dmitrijs Sobolevskis \\ Institute of Power Engineering \\ Riga Technical University \\ Riga, Latvia \\ sauhatas@eef.rtu.lv, romans.petricenko@rtu.lv, zane.broka@rtu.lv, karlis.baltputnis@ rtu.lv, dmitrijs.sobolevskis@sep.lv
}

\begin{abstract}
Reservoir inflow forecasting with artificial neural networks is presented in this paper. Different types of ANN input data were considered such as temperature, precipitation and historical water inflow. Performance of the hourly inflow forecasts was assessed based on a case study of a specific hydropower reservoir in Latvia. The results showed that all the approaches had similar prediction errors implying that for optimal hydropower scheduling uncertainties need to be modelled which is also proposed in this study through generation of several forecast realisations in addition to point predictions.
\end{abstract}

\section{Keywords-ANN; forecasting; hydropower; reservoir inflow}

\section{INTRODUCTION}

To participate in an electricity market, power producers submit their bids the day before based on their planned power generation schedule. For power plants that use renewable intermittent energy sources, forecasts of the availability of the respective source are vital for the daily scheduling. When operating hydroelectric power plants (HPP), the producer needs to forecast the availability of water which is particularly true for run-of-river plants and HPPs with relatively small-capacity reservoirs. In our study, we assume that the bids are submitted to the Nord Pool power exchange Elspot market with an hourly resolution and that the power producer is a price-taker. Consequently, the power producer needs hourly water inflow forecasts for optimal generation scheduling. Besides that, price forecasts for the next day are also essential. For both reservoir inflow and electricity market price forecasting we have employed artificial neural networks (ANN). However, in this paper only inflow forecasting is presented and price forecasts will be a subject of our future publications.

Reservoir inflow forecasting has many applications, including flood control, drought management and hydropower generation [1]. Conventional prediction techniques include time series models suggesting that the inflow is auto-correlated and has the same trends over time. A major disadvantage of such approach is the assumption of stationarity of linearity. To overcome the drawbacks of conventional modelling techniques, artificial intelligence data-driven techniques have been developed [1] one of which-artificial neural networks (ANNs) we use for forecasting in our study due to the ability of ANNs to handle complexity and non-linearity. In [2], time seriesbased techniques, ARMA and ARIMA, are compared with

The work presented in this paper has been co-financed by the National Research Program LATENERGI (2014-2017).
ANNs for inflow forecasting and it is concluded that dynamic autoregressive ANN model with sigmoid activity function delivers better forecasts than the ARIMA and ARMA models. Recurrent neural networks outperformed an ARMAX model also in [3]. A valuable advantage of ANNs is their flexibility in integrating time stamping inputs [4].

Though neural networks are considered a "black-box", careful setup of network parameters and inputs is necessary based on experience and engineering judgement and by involving trial and error [4]. In general, ANN inputs can include historical values of the time series of interest, one or more exogenous input parameters and time indices [4].

In [5], past inflows along with other data such as temperature, precipitation and time-related features is used for reservoir inflow prediction with neural networks. The authors made use of historical records of three years with an hourly resolution by training the ANN on the two-year data and using data of another year for testing. They also experimented with different numbers of hidden neurons to conclude that for a larger number of hidden units the training errors are much lower [5].

In [6], it is concluded that using data on the observed and forecasted precipitation allows to significantly increase the accuracy of inflow forecasts in comparison to time series models that use only the previous observed inflow to form the prediction.

In our study, we have investigated four different types of ANN input data and compared the forecasting performance among them.

\section{METHOD}

We used a three-layer feed-forward ANN and LevenbergMarquardt training algorithm with backpropagation implemented in MATLAB Neural Network Toolbox. Training of the ANN was performed in a supervised manner using historical data as presented in more detail below. The output of ANN provided point forecasts which were further processed by time series smoothing techniques and by imposing prediction residuals of the previous forecasts. The latter approach allowed us to generate additional realizations of the forecast to model the stochasticity of reservoir inflow while not increasing computational burden too much for a practical application to daily operation optimisation of power plants. 


\section{A. Forecasting Approach}

Forecasting of inflow to the HPP reservoir was performed using different types of input data for training of the ANN and prediction. Various approaches were studied to compare their accuracy and choose the best one to be further used for HPP scheduling optimisation. The specific reservoir belongs to the first power plant of an HPP cascade located on the River Daugava. The cascade consists of three run-of-river HPPs with poundage (total installed power $1559.5 \mathrm{MW}$ and total useful volume of reservoirs 215.1 million $\mathrm{m}^{3}$ ). Only the inflow to the first reservoir (main inflow, Fig. 1) needs to be predicted, since the discharge through the upstream power plant determines the inflow to the respective downstream plant and the relatively small lateral inflow to HPP2 and HPP3 is assumed to be constant.

The following approaches were used with different input data to the ANN:

- method (1): historical temperature data and respective reservoir inflow for training of the ANN and temperature forecast for the next day to predict the inflow for that day;

- method (2): historical data of precipitation (particularly, in Daugavpils city located about $150 \mathrm{~km}$ upstream the river from the HPP1) and respective reservoir inflow for training of the ANN and next day precipitation forecast for prediction of the inflow;

- method (3): output data of the approach (1) was used for training of the ANN along with the actual inflow, thus making the ANN learn from the previous forecast errors. For prediction, output of the forecast (1) was used, obtaining another (proposedly improved) forecast for the same day;

- method (4): an existing reservoir inflow forecast was used as input data for training of the ANN along with the corresponding historical inflow data. The specific approach for the existing forecast is not known in detail, but the forecast data is available (this forecast is currently used by the power producer who operates the HPP cascade; further referred to as "conventional forecast"). For prediction, the forecasted inflow of the conventional forecast was used as input data assuming that this forecast would be available at all times and it could be improved by employing ANNs.

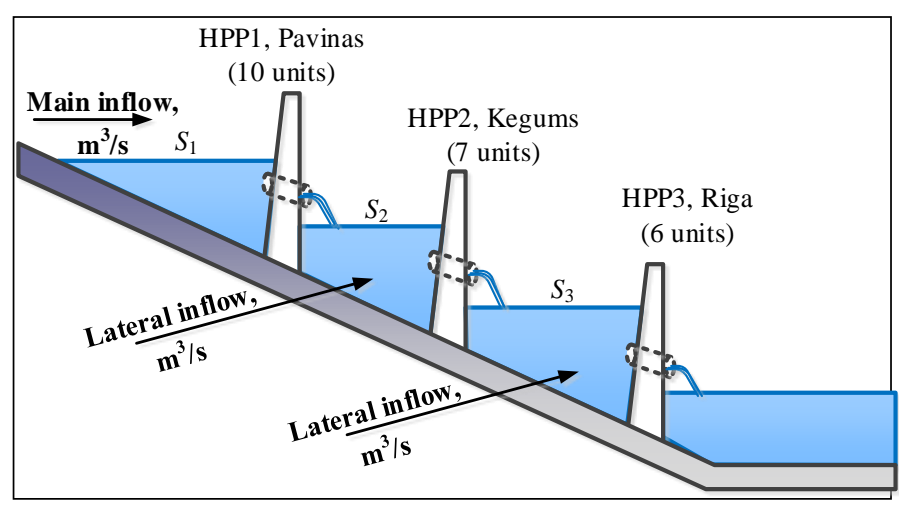

Fig. 1. The cascade of HPPs on the River Daugava
The aim for employing various approaches was to develop an automated forecasting approach in comparison to the conventional forecast which is obtained in a labour-intensive empirical way. Another objective was to derive an improved forecast as compared to the conventional one used by the power producer. Initially, we hypothesized that the forecast (3) or (4) should provide the best results by training on the previous forecast errors.

All the data for training and forecasting was with an hourly resolution. Training data of 2 to 4 previous weeks was employed (the exact amount of data for training was selected randomly). This duration of training was chosen due to the high seasonal and annual variability of river inflow. Additionally, employment of such a period was computationally affordable. As indicated in [7], very large training sets should not be used to avoid overtraining during the learning process.

\section{B. Selection of the ANN parameters}

We designed an algorithm to specify the most suitable parameters of the ANN (Fig. 2), namely, the length of the training data set, $T_{T}$, and the number of neurons in the hidden layer, $N_{H}$. A partial enumeration was performed by randomly selecting the above parameters within the specified limits ( $T_{T}$ from 336 to 672 hours and $N_{H}$ from 10 to 15 neurons) to evaluate the forecast performance for the previous day with the selected parameters. The minimum prediction error of the forecasts for the previous period was used as the criterion for selecting the most suitable ANN parameters for the next forecast horizon. Competing forecasts made by ANNs with differing parameters were compared to the actual observed data and assessed by means of an error measure, namely the root square error: $R M S E=\sqrt{1 / T_{t=1}^{T}\left(y_{t} \hat{y}_{t}\right)^{2}}$, where $\hat{y}_{t}$ are the point forecasts made for the previous forecast period $T=24$ hours, and $y_{t}$ are the corresponding actual observations. After such a partial enumeration, the ANN parameters that provided the least RMSE were chosen for the next forecast horizon. This procedure is repeated for each day of the forecast.

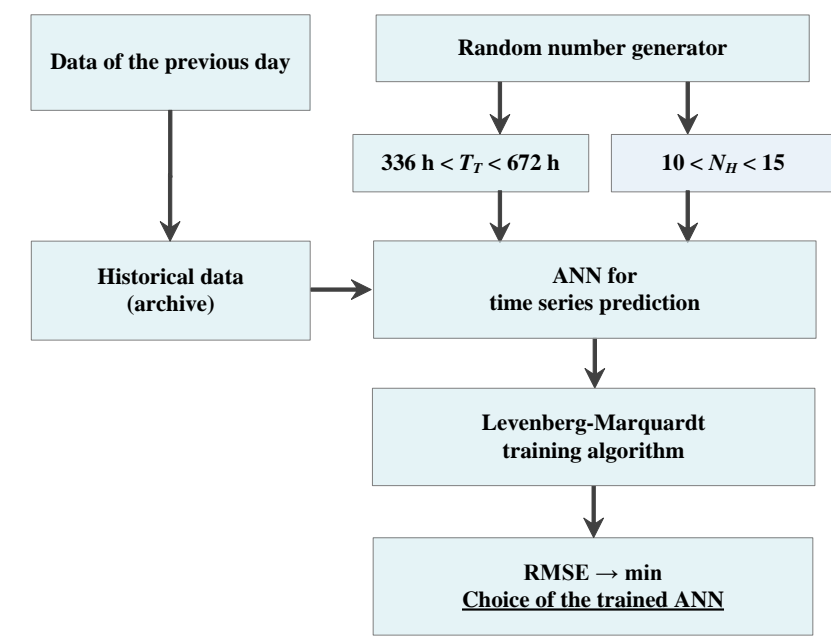

Fig. 2. A simplified diagram of the algorithm for choosing the ANN parameters 


\section{Forecast Smoothing and Modelling of Uncertainties}

The output of ANN provides point forecasts of the hourly water inflow. These forecasted time series were smoothed by calculating the weighted double-sided moving average of different lengths:

$$
\begin{aligned}
& \hat{y}_{t \text { smoothed }}=\hat{y}_{t} \quad \text { for } t \in\{1, T\} ; \\
& \hat{y}_{t \text { smoothed }}=\frac{\hat{y}_{t-1}+\hat{y}_{t}+\hat{y}_{t+1}}{3} \text { for } t \in\{2, T-1\} ; \\
& \hat{y}_{t \text { smoothed }}=\frac{0.5 \cdot\left(\hat{y}_{t-2}+\hat{y}_{t+2}\right)+0.7 \cdot\left(\hat{y}_{t-1}+\hat{y}_{t+1}\right)+\hat{y}_{t}}{3.4} \quad \text { for } t \in[3, T-2],
\end{aligned}
$$

where $\hat{y}_{t \text { smoothed }}$ is the smoothed forecast and $T$ is the forecast horizon (24 hours for forecast smoothing).

Additionally, to take into account uncertainties, we used historical forecast residuals to generate additional realisations of the forecast. By assuming that the forecast errors retain generally the same characteristics in the medium-term, we used the hourly relative errors from the forecasts since 10 days before. Each realisation was obtained by adding or subtracting the historical error to the new forecast at the respective hour. In such a way, the 10-day old historical data provided 20 realisations in addition to the one initial point forecast. We assumed that all the realisations have equal probabilities. Consequently, we obtained 21 realisations of electricity market prices and water inflow which are to be used for stochastic optimisation of power plant operation.

The hourly forecasts presented in next section were evaluated by calculating the mean absolute percentage error and the root mean squared percentage error:

$$
\begin{gathered}
M A P E=\frac{100}{T}_{t=1}^{T}\left|\frac{y_{t} \hat{y}_{t}}{y_{t}}\right| ; \\
R M S P E=100 \cdot \sqrt{\frac{1}{T} \sum_{t=1}^{T}\left(\frac{y_{t}-\hat{y}_{t}}{y_{t}}\right)^{2}} .
\end{gathered}
$$

\section{RESULTS AND DISCUSSION}

We performed forecasts of reservoir inflow using the four different approaches for the period from April 1, 2015 until October 31, 2015. The observed inflow during this period is shown in Fig. 3. During April the inflow was the highest due to spring floods and then sharply dropped from the end of April until June. This is typical for the particular hydropower reservoir and illustrates the large seasonal fluctuations of inflow. Besides that, the inflow is subject to significant annual variations.

Table I summarises the mean errors for all the forecasts including the conventional forecast, whereas Fig. 4-6 illustrate randomly selected examples of hourly forecasts for April 1-7, May 1-7 and September 1-7, 2015, respectively. Forecasting errors for the same time periods are presented in Table II-IV.

The solid blue line in Fig. 4-Fig. 6 shows the observed inflow and the dotted red line presents the conventional forecast. The dashed lines illustrate forecasts we obtained with ANNs using four different types of input data as described before: (1) ambient temperature; (2) precipitation data; (3) forecast (1) used as input; (4) conventional forecast was used as input. It should be noted that the conventional forecast for a single day is provided in the form of a linear function with constant coefficients for each day, thus forming a broken line over several days.

On April 1-7, forecasting approach (3) provided the best results, whereas on May 1-7 the conventional forecast outperformed the ANN forecasts and on September 1-7 the least error was from the forecasting approach (2) which was based on precipitation forecast. Thus our hypothesis that the forecasting approaches (3) and (4) should perform better was true only on May 1-7.

Performance indicators were mostly consistent and showed equal results using the MAPE and RMSPE error measures almost in all cases. However, it is obvious that RMSPE was always larger than the respective MAPE which is because the RMSPE penalises large errors more by squaring them. As shown in Fig. 4-6, the hourly deviations can be significant.

As can be seen in the charts, all the forecasts obtained with ANNs have a similar performance and alike deviations from the observed inflow which is also reflected by errors in Table II-IV.

On April 1-7, the ANN forecasts mostly under-forecasted the inflow, whereas on May 1-7 the same forecasts usually over-forecasted. This can be explained by the fact that data of the previous 2-4 weeks was used for training and the inflow was rapidly increasing at the beginning of April and sharply decreasing in May. Since training was carried out with the previous data when inflow was significantly less or more than during the forecast horizon, the ANN might tend to respectively under-forecast or over-forecast the inflow during the transition period when the spring flooding is starting or ending. In contrast, forecasts for September 1-7 mostly form an average approximation of the actual inflow.

At the meantime, it should also be noted that the observed inflow is subject to measurement errors since it is obtained by integrating measurements over 24 hours and then calculating the hourly inflow.

On September 1-7, inflow forecast errors were 2-3 times larger than in April and May. Partly, it can be explained by larger relative inflow measurement errors, since the measuring system is more suitable for larger inflows (as seen in Fig. 3, the inflow in spring is even 10 times larger than during the rest of year).

The actual inflow is also influenced by small HPPs in Belarus upstream on the River Daugava. Operational data of these HPPs is not available, so it cannot be accounted for in our forecasts.

For HPP scheduling optimisation, the inflow is converted to water head and, additionally, several forecast realisations are considered thus taking into account the uncertainty of forecasts. Consequently, the influence of the aforementioned errors on the optimisation is less than the actual error measures for single-point forecasts. 


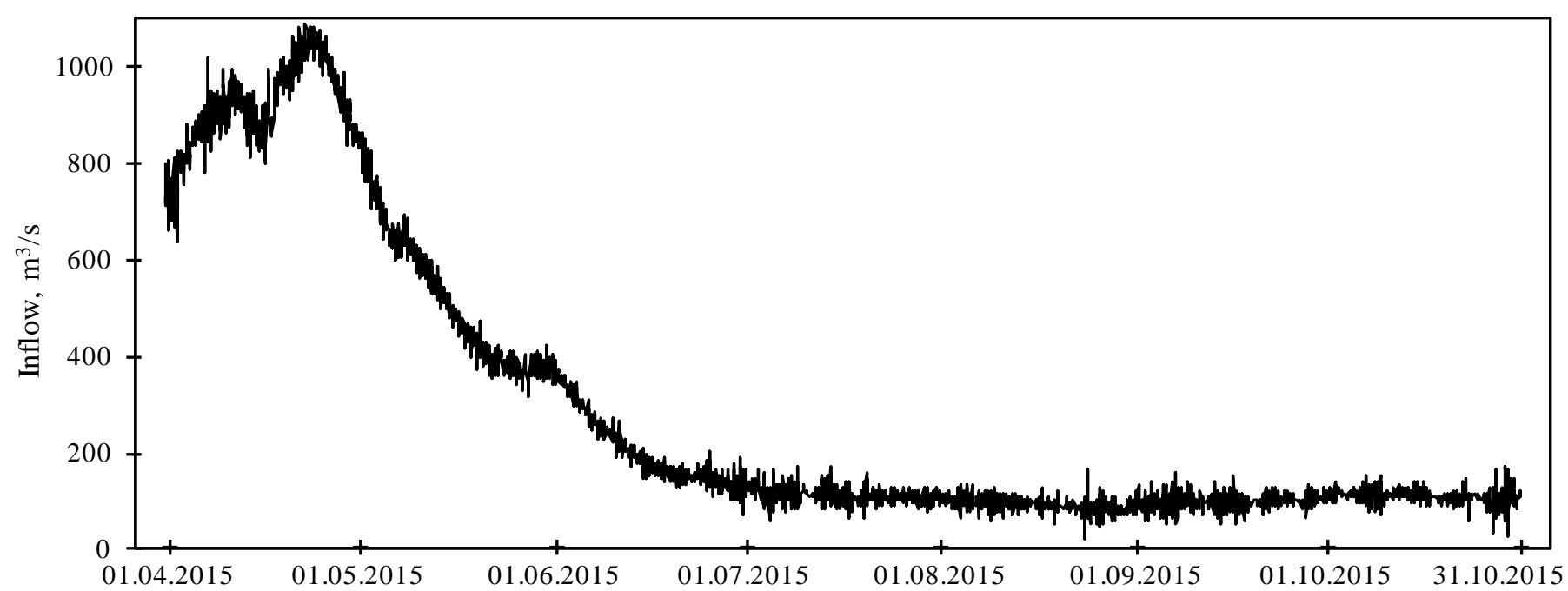

Fig. 3. Observed inflow on April 1-October 31, 2015

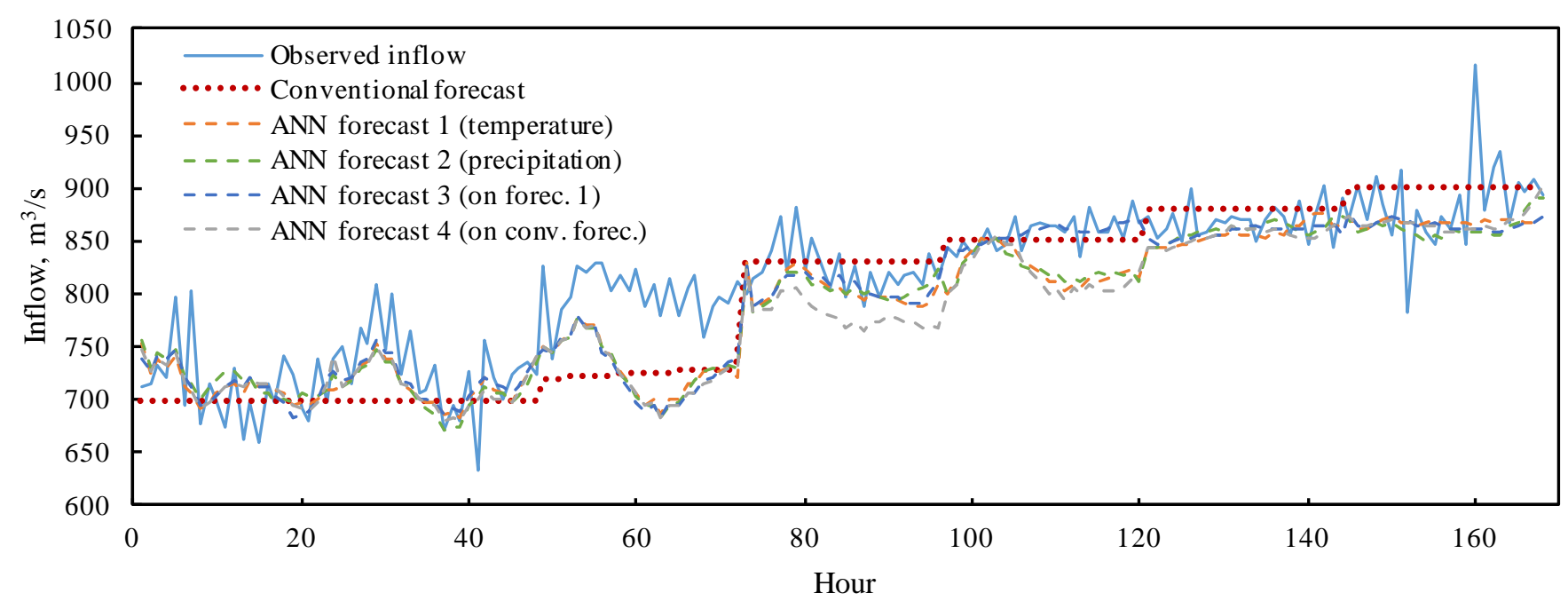

Fig. 4. Forecasted and observed inflow on April 1-7, 2015

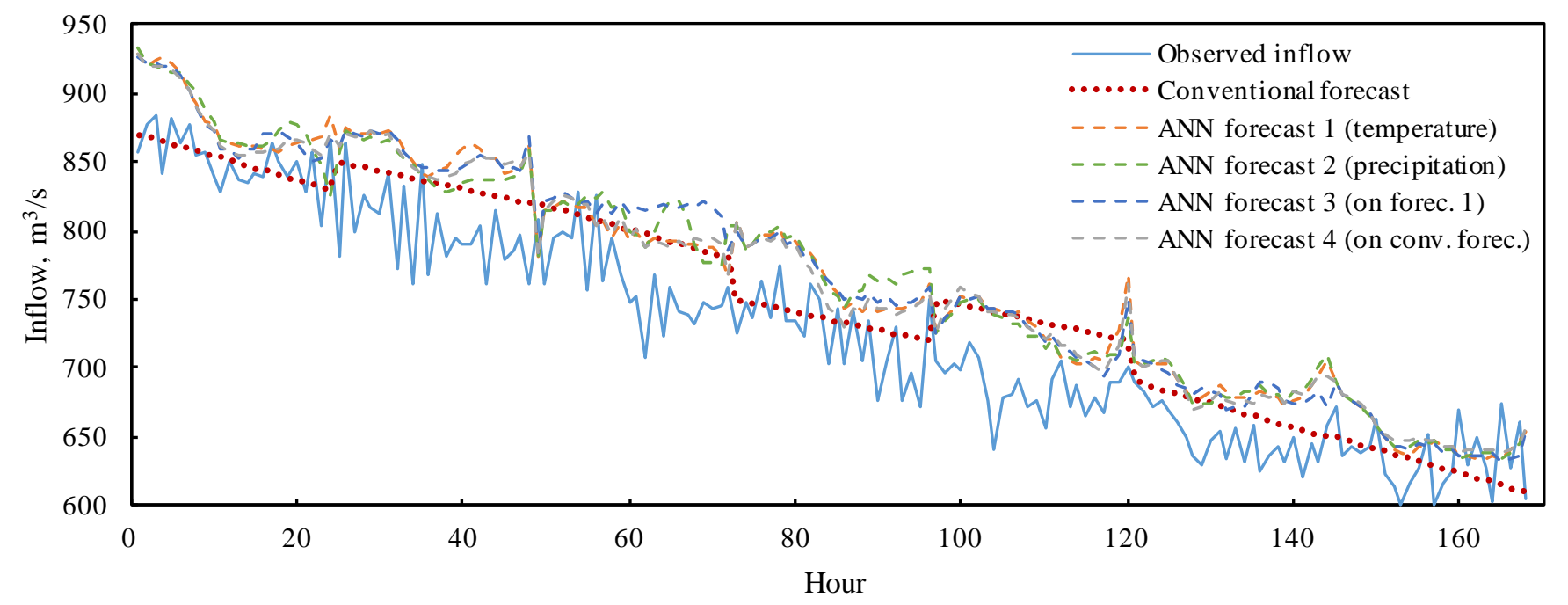

Fig. 5. Forecasted and observed inflow on May 1-7, 2015 


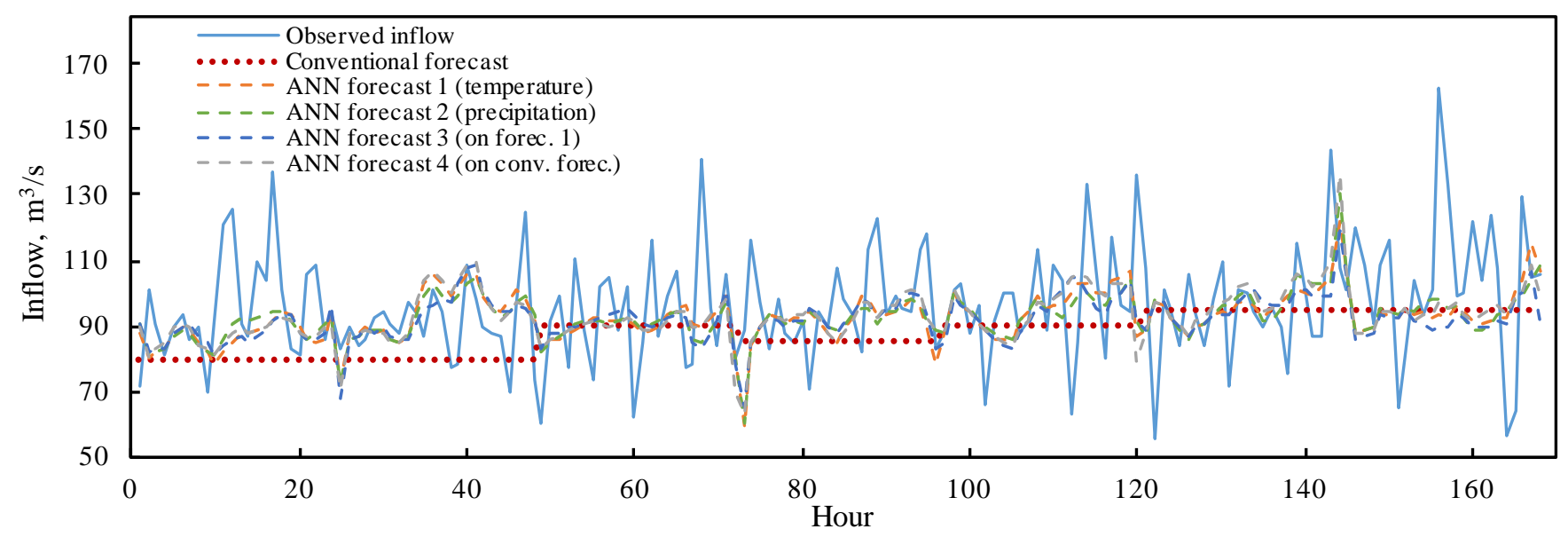

Fig. 6. Forecasted and observed inflow on September 1-7, 2015

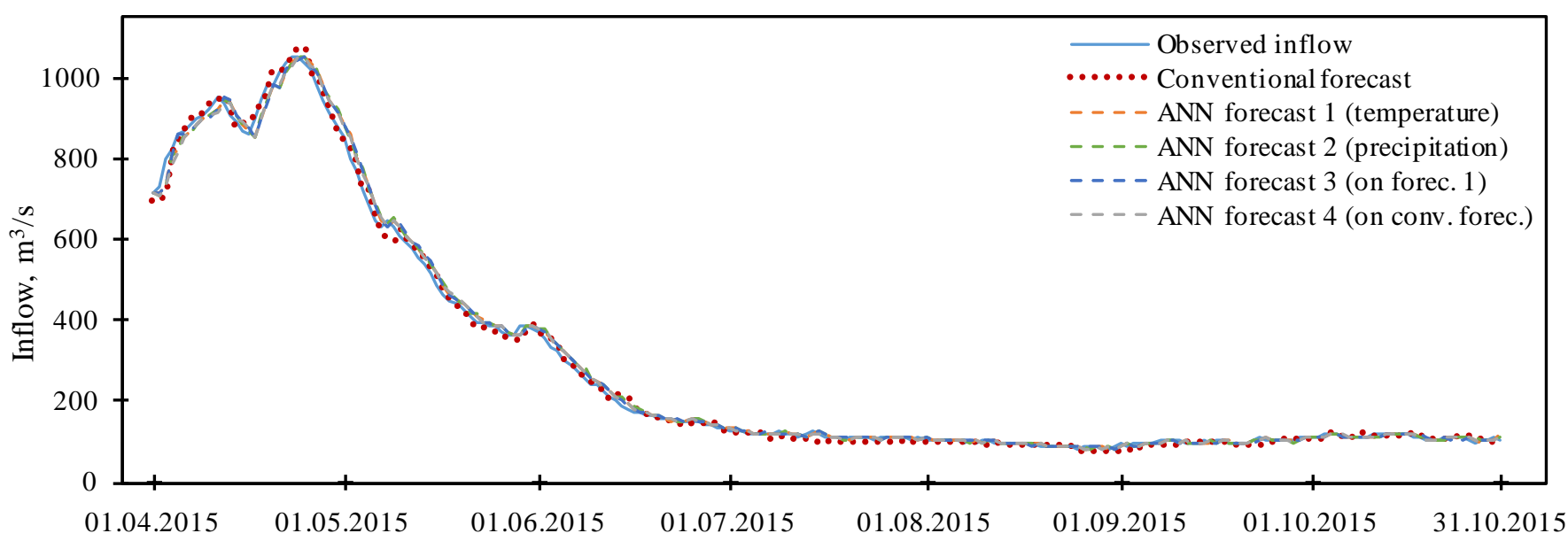

Fig. 7. Average daily forecasted and observed daily inflow on April 1-October 31, 2015

TABLE I. INFLOW FORECAST ERRORS (APRIL 1-OCTOBER 31, 2015)

\begin{tabular}{|l|c|c|}
\hline \multirow{2}{*}{\multicolumn{1}{|c|}{ Forecasting approach }} & \multicolumn{2}{c|}{ Forecast error } \\
\cline { 2 - 3 } & $\boldsymbol{M A P E}$ & $\boldsymbol{R M S P E}$ \\
\hline ANN forecast 1 (temperature) & $7.81 \%$ & $13.16 \%$ \\
\hline ANN forecast 2 (precipitation ) & $7.83 \%$ & $13.12 \%$ \\
\hline ANN forecast 3 (on forecast 1) & $7.81 \%$ & $13.11 \%$ \\
\hline ANN forecast 4 (on conv. forecast) & $7.88 \%$ & $13.29 \%$ \\
\hline Conventional forecast & $\mathbf{7 . 7 0 \%}$ & $\mathbf{1 3 . 0 8 \%}$ \\
\hline
\end{tabular}

TABLE III. INFLOW ForECAST ERRORS (MAY 1-7, 2015)

\begin{tabular}{|l|c|c|}
\hline \multirow{2}{*}{\multicolumn{1}{c|}{ Forecasting approach }} & \multicolumn{2}{c|}{ Forecast error } \\
\cline { 2 - 3 } & $\boldsymbol{M A P E}$ & $\boldsymbol{R M S P E}$ \\
\hline ANN forecast 1 (temperature) & $5.45 \%$ & $6.21 \%$ \\
\hline ANN forecast 2 (precipitation ) & $5.57 \%$ & $6.35 \%$ \\
\hline ANN forecast 3 (on forecast 1) & $5.64 \%$ & $6.49 \%$ \\
\hline ANN forecast 4 (on conv. forecast) & $5.28 \%$ & $6.06 \%$ \\
\hline Conventional forecast & $\mathbf{3 . 9 5 \%}$ & $\mathbf{4 . 9 2 \%}$ \\
\hline
\end{tabular}

TABLE II. INFLOW FORECAST ERRORS (APRIL 1-7, 2015)

\begin{tabular}{|l|c|c|}
\hline \multirow{2}{*}{\multicolumn{1}{|c|}{ Forecasting approach }} & \multicolumn{2}{c|}{ Forecast error } \\
\cline { 2 - 3 } & MAPE & $\boldsymbol{R M S P E}$ \\
\hline ANN forecast 1 (temperature) & $4.07 \%$ & $5.30 \%$ \\
\hline ANN forecast 2 (precipitation ) & $4.08 \%$ & $5.35 \%$ \\
\hline ANN forecast 3 (on forecast 1) & $\mathbf{3 . 6 2 \%}$ & $\mathbf{5 . 1 2 \%}$ \\
\hline ANN forecast 4 (on conv. forecast) & $4.50 \%$ & $5.70 \%$ \\
\hline Conventional forecast & $3.96 \%$ & $5.37 \%$ \\
\hline
\end{tabular}

TABLE IV. INFLOW FORECAST ERRORS (SEPTEMBER 1-7, 2015)

\begin{tabular}{|l|c|c|}
\hline \multirow{2}{*}{\multicolumn{1}{|c|}{ Forecasting approach }} & \multicolumn{2}{c|}{ Forecast error } \\
\cline { 2 - 3 } & MAPE & $\boldsymbol{R M S P E}$ \\
\hline ANN forecast 1 (temperature) & $14.06 \%$ & $19.25 \%$ \\
\hline ANN forecast 2 (precipitation ) & $\mathbf{1 3 . 6 6 \%}$ & $\mathbf{1 8 . 8 1 \%}$ \\
\hline ANN forecast 3 (on forecast 1) & $14.06 \%$ & $19.28 \%$ \\
\hline ANN forecast 4 (on conv. forecast) & $14.21 \%$ & $19.48 \%$ \\
\hline Conventional forecast & $14.76 \%$ & $19.28 \%$ \\
\hline
\end{tabular}




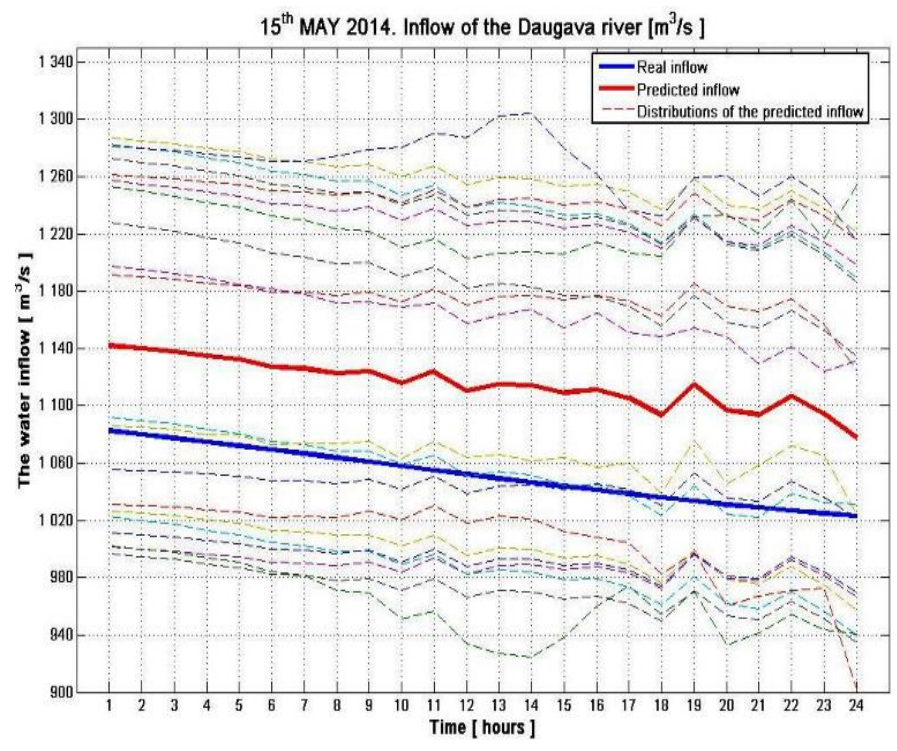

Fig. 8. Forecast realisations of water inflow and the actual inflow

Fig. 7 shows the average daily inflow forecasts and the observed inflow demonstrating that when averaged all the forecasts are close to the actual inflow. It implies that the power production scheduling errors are less if looked at a daily resolution. However, hourly inflow is also important, since the power production bids are based on that, thus influencing the efficiency of power plants and the revenues from sold energy. Therefore we generated additional realisations of forecasts (Fig. 8) as described in section II. All of these prediction realisations are to be taken into account during optimal HPP scheduling to account for uncertainties.

To achieve a better forecasting performance, further analysis of the most suitable ANN structure and parameters as well as input data should be carried out involving large number of trial and error work as suggested in [1] an [5]. Additional data cleansing and pre-processing might also improve the forecasts.

\section{CONCLUSION}

Prediction of reservoir inflow by artificial intelligence techniques allows to automate the forecasting process in comparison to labour-intensive empirical methods. Artificial neural networks with four different types of input data provided a satisfactory accuracy of hydropower reservoir inflow forecasts; however, additional studies of different network structures and parameters as well as input data selection are necessary to improve predictions.

Since the hourly forecasts are subject to uncertainty, it can be tackled by considering several realisations of the predicted inflow which are to be used in stochastic optimisation of HPP scheduling.

\section{REFERENCES}

[1] V. Jothiprakash and R. B. Magar, "Multi-time-step ahead daily and hourly intermittent reservoir inflow prediction by artificial intelligent techniques using lumped and distributed data," J. Hydrol., vol. 450-451, pp. 293-307, 2012.

[2] M. Valipour, M. E. Banihabib, and S. M. R. Behbahani, "Comparison of the ARMA, ARIMA, and the autoregressive artificial neural network models in forecasting the monthly inflow of Dez dam reservoir," $J$. Hydrol., vol. 476, pp. 433-441, 2013.

[3] P. Coulibaly and F. Anctil, "Real-time short-term natural water inflows forecasting using recurrent neural networks," in Neural Networks, 1999 IJCNN '99. International Joint Conference on, 1999, vol. 6, pp. 3802 3805 .

[4] S. I. Vagropoulos, E. G. Kardakos, C. K. Simoglou, A. G. Bakirtzis, and J. P. S. Catalão, "ANN-based scenario generation methodology for stochastic variables of electric power systems," Electr. Power Syst. Res. vol. 134, pp. 9-18, May 2016.

[5] C. Subramanian, M. T. Manry, and J. Naccarino, "Reservoir inflow forecasting using neural networks," in Proceedings of the 61st American Power Conference, 1999, pp. 237-241.

[6] W. Collischonn, C. E. Morelli Tucci, R. T. Clarke, S. C. Chou, L. G Guilhon, M. Cataldi, and D. Allasia, "Medium-range reservoir inflow predictions based on quantitative precipitation forecasts," J. Hydrol., vol. 344, no. 1-2, pp. 112-122, Sep. 2007.

[7] J. P. S. Catalão, S. J. P. S. Mariano, V. M. F. Mendes, and L. a F. M. Ferreira, "Short-term electricity prices forecasting in a competitive market: A neural network approach," Electr. Power Syst. Res., vol. 77, no. 10 , pp. $1297-1304,2007$.

Antans Sauhats received Dipl.Eng., Cand.Techn.Sc. and Dr.Hab.Sc.Eng. degrees from Riga Technical University (former Riga Polytechnical Institute) in 1970, 1976 and 1991 respectively. Since 1991, he is Professor of Electric Power Systems and since 1996, he is Director of the Institute of Power Engineering of RTU.

Roman Petrichenko received B.Sc., M.Sc. and Dr.Sc.Eng. degrees in Electrical Engineering from Riga Technical university in 2006, 2008 and 2014 respectively. His research interests include power system automation and optimisation. Since 2014, he is a Leading Researcher of the Institute of Power Engineering Institute of RTU.

Zane Broka received her Master's degree in Power Engineering from Riga Technical University in 2014. Currently, she is a 3rd year PhD Researcher at the Institute of Power Engineering of RTU. Her major field of study is power system optimisation. Research interests also include terminology development and standardisation.

Karlis Baltputnis received his Master's degree in Power Engineering from Riga Technical University in 2015. Presently, he is a Research Assistant and a $2 \mathrm{nd}$ year PhD student at the Institute of Power Engineering of RTU. His main research interests are in generation scheduling optimisation, hydrothermal dispatch and energy storage.

Dmitrijs Sobolevskis received B.Sc. and M.Sc degrees in Power and Electrical Engineering from Riga Technical University in 2007 and 2009 respectively. Since 2009, he is an Electrical Engineer in JSC "Siltumelektroprojekts". Since 2009, he is a PhD student and since 2014, Researcher of the Institute of Power Engineering of RTU. General scientific activities are Latvian energy system modelling and optimisation. 